\title{
The Hippocamposeptal Pathway Generates Rhythmic Firing of GABAergic Neurons in the Medial Septum and Diagonal Bands: An Investigation Using a Complete Septohippocampal Preparation In Vitro
}

\author{
Frédéric Manseau, Romain Goutagny, Marc Danik, and Sylvain Williams \\ Douglas Hospital Research Center, Department of Psychiatry, McGill University, Montreal, Quebec, Canada H4H 1R3
}

\begin{abstract}
The medial septum diagonal band area (MS/DB) projects to the hippocampus through the fornix/fimbria pathway and is implicated in generating hippocampal theta oscillations. The hippocampus also projects back to the MS/DB, but very little is known functionally about this input. Here, we investigated the physiological role of hippocamposeptal feedback to the MS/DB in a complete in vitro septohippocampal preparation containing the intact interconnecting fornix/fimbria pathway. We demonstrated that carbachol-induced rhythmic thetalike hippocampal oscillations recorded extracellularly were synchronized with powerful rhythmic IPSPs in whole-cell recorded MS/DB neurons. Interestingly, we found that these IPSPs evoked rebound spiking in GABAergic MS/DB neurons. In contrast, putative cholinergic and glutamatergic MS/DB neurons responded only weakly with rebound spiking and, as a result, were mostly silent during theta-like oscillations. We next determined the mechanism underlying the rebound spiking that followed the IPSPs in MS/DB GABAergic neurons using phasic electrical stimulation of the fornix/fimbria pathway. We demonstrate that the increased rebound spiking was attributable to the activation of $I_{\mathrm{h}}$ current, because it was significantly reduced by low concentrations of the $I_{\mathrm{h}}$ antagonist ZD7288 [4- $(N$-ethyl- $N$ phenylamino)-1,2-dimethyl-6-(methylamino) pyridinium chloride]. Together, these results suggest that rhythmical activity in hippocampus is transferred to the MS/DB and can preferentially phase the spiking of GABAergic MS/DB neurons because of their significant expression of $I_{\mathrm{h}}$ currents. Our data demonstrate that hippocamposeptal inhibition facilitates theta rhythmic discharges in MS/DB GABAergic neurons while favoring the inhibition of most ACh and glutamate neurons.
\end{abstract}

Key words: medial septum; hippocamposeptal feedback; GABAergic neurons; carbachol; rhythmic inhibition; $I_{\mathrm{h}}$ current; theta oscillation

\section{Introduction}

The medial septum diagonal band area (MS/DB) is a key generator of $3-12 \mathrm{~Hz}$ theta oscillations in the hippocampus (Buzsaki, 2002), and theta activity of hippocampal neurons is implicated in spatial cognition, memory processes, and sensorimotor integration (Winson, 1978; Bland and Oddie, 2001; Buzsaki, 2002). Although how the MS/DB generates hippocampal theta is incompletely understood, the current model suggests that MS/DB cholinergic (ACh) and GABAergic neurons have distinct roles. MS/DB ACh neurons may convey a sustained depolarization of hippocampal neurons, whereas MS/DB GABAergic neurons rhythmically inhibit hippocampal GABAergic interneurons, which in turn pace pyramidal neurons (Freund and Antal, 1988; Toth et al., 1997). A role in theta for the recently described glutamate neurons in the MS/DB still remains to be determined

Received Sept. 28, 2007; revised March 12, 2008; accepted March 12, 2008.

This work was supported by the Canadian Institute of Health Research, the Natural Sciences and Engineering Research Council of Canada, and Fonds de la Recherche en Santé du Québec. F.M. was supported by a fellowship from the Alzheimer Society of Canada.

Correspondence should be addressed to Dr. Frederic Manseau, European Brain Research Institute, "Rita LeviMontalcini" Foundation, Via del Fosso di Fiorano, 64/65, 00143 Rome, Italy. E-mail: f.manseau@ebri.it.

DOI:10.1523/JNEUROSCI.0247-08.2008

Copyright $\odot 2008$ Society for Neuroscience $\quad$ 0270-6474/08/284096-12\$15.00/0
(Sotty et al., 2003; Manseau et al., 2005; Colom et al., 2005). Conversely, the hippocampus is known to have important projections back to the MS/DB (Alonso and Kohler, 1982; Toth et al., 1993). The input from hippocampus to septum originates principally from GABAergic neurons located in st. oriens of CA1/CA3 (Toth et al., 1993; Gulyas et al., 2003). These hippocamposeptal projecting GABA neurons are innervated by local CA1/3 pyramidal neurons and are therefore critically situated to transmit rhythmic activity to the septum (Blasco-Ibanez and Freund, 1995). Hippocamposeptal GABA neurons synapse preferentially in the MS/DB on GABAergic neurons but also to a lesser extent on ACh neurons (Toth et al., 1993). In contrast, CA1/CA3 pyramidal neurons may directly innervate only neurons of the lateral septum, which are sparsely connected to the MS/DB (Leranth et al., 1992; Linke et al., 1995). The inhibitory hippocamposeptal pathway is therefore considered the only monosynaptic hippocampal connection back to the MS/DB. Although there is very little physiological evidence about the role of this hippocamposeptal inhibitory pathway on MS/DB activity, it has been suggested that it may inhibit (Hasselmo and Schnell, 1994; Rokers et al., 2002) or pace (Wang, 2002) rhythmic activity in the MS/DB. For example, the role of the hippocampal feedback may be to inhibit ACh neurons and provide an efficient feedback mecha- 
nism for the hippocampus to control ACh levels to perform novelty/familiarity information encoding (Hasselmo and Schnell, 1994; Rokers et al., 2002). However, early physiological investigations have shown that the hippocampal input to the MS/DB is more complex than a simple inhibitory feedback, because fornix/ fimbria stimulation can evoke a variety of effects in MS/DB neurons ranging from inhibition to excitation or both (McLennan and Miller, 1974; Dutar et al., 1985). More recently, Dragoi et al. (1999) showed that MS/DB unit activity can be positively or negatively correlated with hippocampal activity in a state-dependent manner. Our aim was to investigate the physiological role of the rhythmic inhibition originating from hippocampus on MS/DB neurons, to determine whether this rhythmic activity could pace and/or inhibit MS/DB activity, and to assess which MS/DB neuronal types were modulated by hippocamposeptal inhibitory activity. To accomplish this, we used a novel in vitro preparation comprising a complete hippocampus fully connected with a hemiseptum. Our results offer a new perspective on septohippocampal interactions in the generation of theta activity.

\section{Materials and Methods}

Acute septohippocampal preparation. All procedures were performed according to protocols and guidelines approved by the McGill University Animal Care Committee and the Canadian Council on Animal Care. Briefly, young [postnatal day 12 (P12) to P18] Sprague Dawley rats (Charles River Canada, Montreal, Quebec, Canada) were deeply anesthetized with isofluorane and subsequently decapitated. The brain was rapidly removed and placed in ice-cold high-sucrose artificial CSF (ACSF) (Jones et al., 1999) with the following composition (in mM): 252 sucrose, $3 \mathrm{KCl}, 2 \mathrm{MgSO}_{4}, 24 \mathrm{NaHCO}_{3}, 1.25 \mathrm{NaH}_{2} \mathrm{PO}_{4}, 1.2 \mathrm{CaCl}_{2}$, and 10 glucose ( $\mathrm{pH} 7.2$ when bubbled with carbogen). After removal of the cerebellum and frontal part of the brain, a cut was made through the interhemispheric sulcus to separate the two hemispheres thereby exposing the hemisected septum. As described previously (Manseau et al., 2005), the half-septum and hippocampus were then carefully dissected out from each hemisphere by inserting a flat microspatula in the lateral ventricle and sliding along the corpus callosum at both dorsal and ventral contours of hippocampus and septum. The half-septum and hippocampus still attached together by the septohippocampal fibers of the fornix/fimbria bundle were then left to rest in high-sucrose ACSF solution (room temperature) for $1-3 \mathrm{~h}$ before the start of recordings. For all experiments, single septohippocampal preparations were transferred to a fully submerged custom-made Plexiglas chamber (see below) and continuously perfused with ACSF $(2-4 \mathrm{ml} / \mathrm{min}$; gravity fed; room temperature, $\sim 21^{\circ} \mathrm{C}$ ) containing the following (in $\mathrm{mm}$ ): $126 \mathrm{NaCl}, 24 \mathrm{NaHCO}_{3}, 10$ glucose, $3 \mathrm{KCl}, 2 \mathrm{MgSO}_{4}, 1.25 \mathrm{NaH}_{2} \mathrm{PO}_{4}$, and $2 \mathrm{CaCl}_{2}$ (pH 7.4, with $95 \%$ $\mathrm{O}_{2} / 5 \% \mathrm{CO}_{2}$ ). Recordings were only started after an equilibration period of least $30 \mathrm{~min}$. Placement of the preparation in the chamber was such that the MS/DB area of the septum and the CA3 region of the hippocampus were exposed at the surface (see Fig. $2 \mathrm{~A}$ ) allowing direct access for the recording pipettes. Septohippocampal preparations made in the morning could retain good synaptic responses and neuronal properties for several hours. To visualize the location of electrode placement within the MS/DB and hippocampus, we used a Nikon (Tokyo, Japan) microscope (Eclipse E600FN) equipped with $10 \times$ water immersion objective.

Local field potential recording. Extracellular field potentials were recorded conventionally using glass micropipettes (1-3 M $\Omega$ ) filled with normal ACSF. The electrode tip was placed consistently on the inside curve of the intact hippocampus in a region corresponding to CA3 (see Figs. $1 \mathrm{~A}, 2 \mathrm{~A}$ ). The electrodes were lowered into the tissue in or near the pyramidal layer. In control experiments with the isolated intact hippocampus, field recording electrodes were filled with pontamine sky blue (PSB) ( $2 \%$ in $0.5 \mathrm{M} \mathrm{NaCl})$, and PSB was injected iontophoretically (10 $\mu \mathrm{A})$ at the end of the session to localize the recording site. Recordings were performed using a BVC-700A amplifier (Dagan, Minneapolis, MN) equipped with an AC-coupled extracellular Headstage (model 8024) with a $1000 \times V_{\mathrm{m}}$ gain and a low-cut filter set at $0.3 \mathrm{~Hz}$.
Whole-cell recordings and cell characterization. Whole-cell recordings were performed using borosilicate glass pipettes with a resistance of 3-7 $\mathrm{M} \Omega$ when filled with the following (in $\mathrm{mm}$ ): $144 \mathrm{~K}$-gluconate, $3 \mathrm{MgCl}_{2}$, 0.2 EGTA, 10 HEPES, 2 ATP, and 0.3 GTP, pH 7.2 (285-295 mOsm). Patching in the whole-cell configuration from MS/DB neurons in the septohippocampal preparation was done using the "blind" patch technique (Blanton et al., 1989) by slowly descending the recording pipette into the tissue while monitoring the electrode resistance until a rapid drop was noticed when contact was made with the surface of a cell. All recordings in voltage-clamp and current-clamp modes were performed using a patch-clamp amplifier PC-505A (Warner Instruments, Hamden, CT).

The resting membrane potential was measured in current-clamp mode once a stable recording was obtained. Cells were kept for experiments only when the resting membrane potential was more negative than $-45 \mathrm{mV}$, spikes overshot $0 \mathrm{mV}$, and the series resistance was $<30 \mathrm{M} \Omega$. As described previously (Sotty et al., 2003; Manseau et al., 2005), the firing properties of each neuron were assessed using a series of depolarizing and hyperpolarizing current pulses $(0-200 \mathrm{pA} ; 2-8 \mathrm{~s})$ applied from $-60 \mathrm{mV}$. The presence of a voltage-dependent inward rectification (depolarizing sag) in response to series of $2 \mathrm{~s}$ hyperpolarizing current pulses was noted, and its amplitude was measured for the current pulse inducing an initial hyperpolarization to $-95 \mathrm{mV}$. The same pulse was used to determine whether the neuron had intrinsic bursting properties as evidenced by rebound firing. Afterhyperpolarization duration was measured for the first action potential evoked from threshold, and firing frequencies were determined for each neuron using a $2 \mathrm{~s}$ depolarizing current pulse $50 \mathrm{pA}$ greater than that required to depolarize the cell to threshold from $-60 \mathrm{mV}$. Maximal firing frequency was calculated from the time interval between the first and the second spike in a train, steady firing frequency was calculated from the time interval between the last two spikes in a train, and mean firing rate was calculated from the number of spikes evoked by a $2 \mathrm{~s}$ depolarizing current pulse. Spike accommodation was measured by calculating the following for each cell: (maximal firing frequency - steady-state firing frequency)/maximal firing frequency. Individual cells were sorted into four categories according to previously established electrophysiological criteria (Griffith and Matthews, 1986; Gorelova and Reiner, 1996; Jones et al., 1999; Sotty et al., 2003; Manseau et al., 2005): (1) slow-firing neurons shown previously to be cholinergic (Griffith and Matthews, 1986; Sotty et al., 2003; GarridoSanabria et al., 2007) displayed a low firing frequency during depolarization and no inward rectification during hyperpolarization ( $I_{\mathrm{h}}$ current), (2) fast-firing neurons, and (3) burst-firing neurons, shown previously to be GABAergic (Knapp et al., 2000; Sotty et al., 2003; Morris et al., 2004), showed a strong $I_{\mathrm{h}}$-mediated inward rectification (depolarizing "sag") in response to hyperpolarizing steps. Fast-firing cells typically fired tonically during subthreshold depolarization. Burst-firing neurons displayed higher $F_{\mathrm{MAX}}$ values and stronger spike frequency accommodation and produced prominent rebound bursts after hyperpolarizing current pulses. Finally, (4) cluster-firing neurons, which have been identified previously as glutamate neurons within the MS/DB (Sotty et al., 2003), displayed clusters of closely spaced action potentials separated by short intervals of subthreshold membrane oscillations during long-lasting depolarizations. A minority of cells could not be identified clearly using these criteria, because they showed mixed properties and were therefore not included in statistics. Table 1 summarizes the properties of cells belonging to the four main groups.

For all voltage recordings (patch and field), signals were continuously low-pass filtered on-line at $1-2 \mathrm{kHz}$ and sampled at $10-20 \mathrm{kHz}$ using pClamp ( 9 and 10) software (Molecular Devices, Sunnyvale, CA). Traces were additionally filtered off-line for analysis and presentation. Recorded membrane potentials were not corrected for junction potentials (estimated at $-14.4 \mathrm{mV}$ ).

Measurement of carbachol-induced activity. Continuous recordings were performed simultaneously from the hippocampus (extracellular field) and from the MS/DB (patch), whereas the muscarinic agonist carbachol $(\mathrm{CCH})(15-30 \mu \mathrm{M})$ was used to induce activity in septohippocampal preparations. Although carbachol-induced activity was variable and occurred in relatively short episodes, it was often possible to repeat the 
Table 1. Firing characteristics of electrophysiologically identified MS/DB neurons receiving rhythmic inhibitory input from the hippocampus

\begin{tabular}{|c|c|c|c|c|}
\hline & Slow firing & Fast firing & Burst firing & Cluster firing \\
\hline Carbachol & $n=3$ & $n=6$ & $n=11$ & $n=4$ \\
\hline Stimulations (fornix/fimbria) & $n=20$ & $n=9$ & $n=31$ & $n=12$ \\
\hline Total recorded cells & $n=23$ & $n=15$ & $n=46$ & $n=16$ \\
\hline$V_{\mathrm{m}}$ rest & $-53.6 \pm 1.3$ & $-54.1 \pm 2.1$ & $-53.3 \pm 0.9$ & $-51.2 \pm 1.3$ \\
\hline$F_{\text {MAX }}(\mathrm{Hz})$ & $6.5 \pm 0.8$ & $13.9 \pm 1.6$ & $21.3 \pm 2.0(\mathrm{~s}, \mathrm{f}, \mathrm{c})$ & $8.9 \pm 1.1$ \\
\hline$F_{\text {STEADY }}(\mathrm{Hz})$ & $1.1 \pm 0.3$ & $8.5 \pm 0.5(s, b, c)$ & $4.0 \pm 0.5(\mathrm{~s})$ & $4.7 \pm 0.7(s)$ \\
\hline Accommodation (\%) & $84.6 \pm 4.1(f, c)$ & $30.3 \pm 6.1$ & $78.7 \pm 2.0(\mathrm{f}, \mathrm{c})$ & $44.3 \pm 6.3$ \\
\hline Mean firing rate $(\mathrm{Hz})$ & $3.9 \pm 0.6$ & $18.7 \pm 1.2(s, b, c)$ & $9.6 \pm 1.0(\mathrm{~s})$ & $8.8 \pm 1.2(\mathrm{~s})$ \\
\hline AHP duration (ms) & $257.3 \pm 23.7(b)$ & $203.1 \pm 33.2(b)$ & $94.2 \pm 13.8$ & $302.8 \pm 24.3(\mathrm{f}, \mathrm{b}$ \\
\hline Amplitude sag (mV) & $1.5 \pm 0.5$ & $7.0 \pm 0.8(s)$ & $6.7 \pm 0.6(\mathrm{~s})$ & $4.6 \pm 0.7(s)$ \\
\hline
\end{tabular}

For analysis of intrinsic cell properties, an ANOVA with post hoc Tukey's multiple comparison test was used to compare the different groups. Significant differences (at least $p<0.05$ ) are marked: $s$, slow-firing neurons; $\mathrm{f}$, fast-firing neurons; b, burst-firing neurons; $c$, cluster-firing neurons. Note that values for each cell type are pooled across three different experimental conditions and that a small number of cells receiving IPSPs from the hippocamposeptal pathway could not be included, because their characterization was incomplete or their intrinsic properties did not allow unambiguous classification.

experiment several times with a single preparation after an adequate washing out of carbachol ( $\sim 15 \mathrm{~min})$. The rhythmic events induced by carbachol in hippocampus were prominent at frequencies of $1-5 \mathrm{~Hz}$.

Postinhibitory-rebound spike analysis. To describe the cellular response of MS/DB neurons to carbachol application, we initially examined changes in membrane potential and bulk firing frequency during relatively long control and carbachol recording periods (1 min). However, to get a more accurate quantification of the effect of IPSPs on MS/DB cell firing, we analyzed data collected over short periods (20 s epochs), where carbachol-induced hippocampal activity was stable and maximal and selected a subset of experiments in which a clearly regular pattern of extracellular hippocampal activity and synchronized MS/DB IPSPs were present. We counted "rebound" spikes for which the initiation phase was clearly continuous with the repolarization phase of IPSPs (action potential within $200 \mathrm{~ms}$ of $V_{\mathrm{m}}$ repolarization to its level just before the IPSP). This method allowed spontaneous spikes occurring between distant nonrhythmic IPSPs to be rejected and spikes that were clearly part of the same rebound burst (doublets or triplets) to be counted. Unless otherwise mentioned, all event correlation analysis was done by comparing the time of peak (maximum positive voltage deflection) of individual hippocampal field potentials against IPSP onset and rebound spikes in MS/DB neurons.

Dual-bath chamber experiments. A custom-made recording chamber was used to allow optimal perfusion of the intact septohippocampal preparation in our experiments. In this chamber, the tissue was placed and weighted down on a nylon mesh, which was suspended $2 \mathrm{~mm}$ from the bottom. A Teflon wall was then inserted between the septum and hippocampus at the level of the fornix/fimbria bundle so that the two structures could be perfused independently with ACSF containing different pharmacological agents (see Fig. $2 A$ ). This wall was sealed with inert grease but had a small gap just large enough to accommodate the fornix/fimbria bundle without disconnecting the fibers. In every dual chamber experiment, we checked that the two compartments were isolated from each other by stopping the suction on one side and checking that the level would rise on this side only. We also checked for electrical isolation of the two compartments by verifying that the recording electrode on one side of the bath was not grounded properly when its reference electrode was in the other chamber. Finally, in some experiments, we also perfused bicuculline either in septum or hippocampus and found that only when bicuculline was applied to the hippocampus did it trigger the well recognized interictal-like activity further demonstrating the complete separation of the two compartments.

Electrical stimulation. Compound inhibitory synaptic events were evoked using short duration, low intensity constant current stimuli [50$150 \mu \mathrm{A}$; five pulse of $0.5 \mathrm{~ms}$ duration; interstimulus interval (ISI), $10 \mathrm{~ms}$ ] delivered via a monopolar tungsten microelectrodes (World Precision Instruments, Sarasota, FL) placed in the fornix/fimbria bundle and connected to a constant current Isolated Pulse Stimulator (A-M Systems, Sequim, WA) (for an illustration of the experimental setup, see Fig. 6A). Although stimulation in the hippocampus proper rarely evoked synaptic response in MS/DB cells, it was found that placement of the electrode tip in the dorsal fornix between the hippocampus and septum elicited reli- able responses. In general, after finding a stimulation site that consistently evoked IPSPs in the recorded MS/DB neuron, different train frequencies $(1-3 \mathrm{~Hz})$ were assessed to find one that could trigger optimal rebound action potentials (i.e., train frequency was adjusted to match with the duration of evoked IPSPs). Once a frequency was selected for a given neuron, we acquired repeated (two to five times, $30 \mathrm{~s}$ ) alternating episodes of baseline (no stimulation) and stimulation conditions.

Materials. Carbachol, bicuculline, glucose, and all inorganic salts were obtained from Sigma (Oakville, Ontario, Canada). All drugs came from aliquots of stock solutions (stored at $-20^{\circ} \mathrm{C}$ ) and added to the perfusing ACSF at the proper concentrations. Pharmacological blockade of $I_{\mathrm{h}}$ was done with ZD7288 [4-(N-ethyl- $N$-phenylamino)-1, 2-dimethyl-6(methylamino)pyridinium chloride] (ZD) (Tocris Cookson, Ellisville, $\mathrm{MO})$.

Data analysis. Electrophysiological data were analyzed and plotted using pClamp, Spike2 (5.12; Cambridge Electronic Design, Cambridge, UK) and Origin (5.0; OriginLab, Northampton, MA). All results are expressed as mean \pm SEM. For statistical analyses, we performed paired or unpaired Student's $t$ test (two-tailed) or ANOVA using Prism (GraphPad Software, San Diego, CA). $p$ values of $<0.05$ were considered to be statistically significant.

\section{Results}

Carbachol-induced hippocampal oscillations synchronize the rhythmic inhibition and firing of MS/DB neurons

Our first aim was to evaluate the role of hippocampal input to the $\mathrm{MS} / \mathrm{DB}$ in the coordination of septohippocampal activity. To this mean, we used a newly developed in vitro septohippocampal preparation combined with local field potential (LFP) and singlecell electrophysiological recordings from the hippocampus and septum. We first accessed whether the cholinergic agonist carbachol, which is well known to produce different types of rhythmic activity in the hippocampus (Konopacki et al., 1987; MacVicar and Tse, 1989; Fisahn et al., 2002; Fellous and Sejnowski, 2000; Colgin et al., 2003), could be used as a pharmacological tool to activate hippocampal oscillations.

As shown in Figure 1, hippocampal network oscillations were effectively triggered by perfusing the intact isolated hippocampus with the cholinergic agonist carbachol $(15-30 \mu \mathrm{M})$. By recording concomitantly field activity with a pipette placed in st. pyramidal of CA3 (Fig. 1 A) and CA3 pyramidal cells with a patch pipette, we found that pyramidal neurons had a high probability to fire one action potential phase locked close to the peak of the field oscillation (spike probability, $\leq 0.3 ; n=5$ ) (Fig. $1 B-D$ ). These experiments suggest that carbachol can evoke rhythmic single spiking of CA3 pyramidal neurons with some characteristics resembling pyramidal cell firing observed during theta in vivo (Soltesz and Deschenes, 1993; Bland et al., 2005) and is thus an adequate method to evoke physiologically relevant oscillatory activity. 


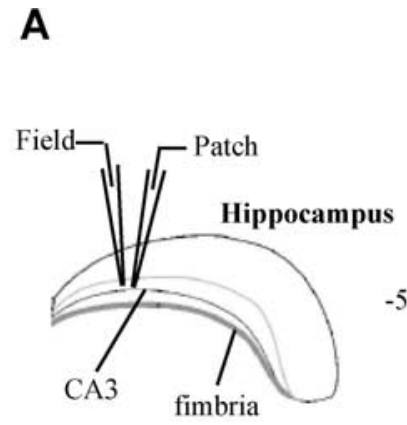

B

E

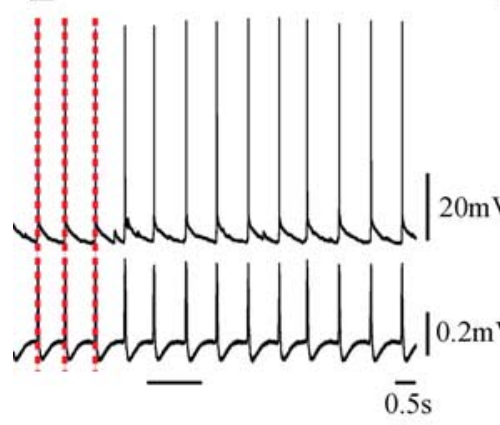

$\mathbf{F}$

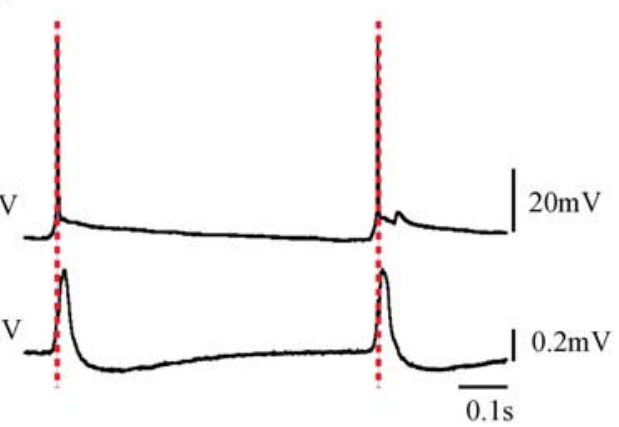

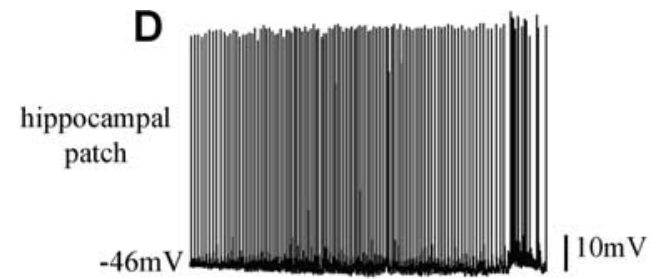

hippocampal
field

G

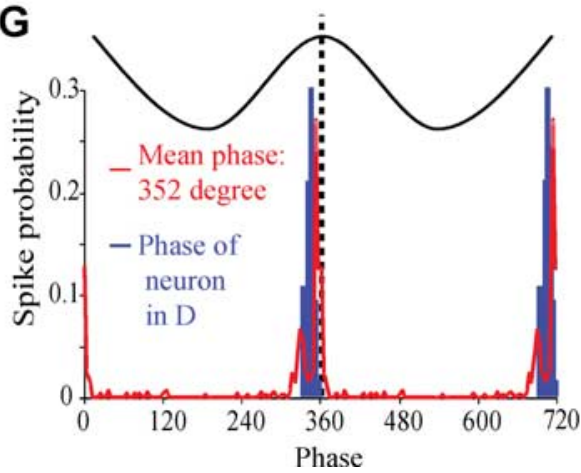

Figure 1. Characterization of the hippocampal oscillations induced by carbachol. $A$, Schematic representation of the recording. Field recording electrode filled with PSB ( $2 \%$ in $0.5 \mathrm{~m}$ NaCl) and patch electrode were lowered into $C A 3$ pyramidal layer. $B$, Electrophysiological properties of the pyramidal neurons recorded. $C$, At the end of the patch recording, PSB was iontophoretically injected $(10 \mu \mathrm{A})$ to localize the recording site. $\mathbf{D}-\mathbf{G}$, The pyramidal neurons fire at the peak of the field oscillation, with a mean phase of $347^{\circ}(\mathbf{G}$, blue line). On average $(\mathbf{G}$, red line), the five cells recorded fired near the peak of the field oscillations.

Higher-frequency events in the gamma frequency bands were sometimes observed (Fisahn et al., 2002) but were not analyzed further.

We then sought to assess whether the oscillatory activity elicited in the hippocampus by carbachol would be associated with rhythmic activity in MS/DB neurons. To specifically investigate the role of the hippocampal input while excluding the direct actions of carbachol in the MS/DB, the septohippocampal preparation was placed in a custom-made dual-bath recording chamber designed to allow independent perfusion of the septum and hippocampus with different pharmacological agents while preserving the connecting fornix/fimbria fibers (for an image of the experimental set-up, see Materials and Methods) (Fig. 2A). Great care was used to assure that the two chambers were sealed from each other (see Materials and Methods). Using this configuration, we performed simultaneous extracellular LFP recordings from CA3 of the hippocampus and whole-cell patch-clamp recordings from MS/DB neurons. When control ACSF was perfused on both hippocampal and septal sides, the extracellular LFP recordings from CA3 displayed little or no evidence of field activity, whereas MS/DB neurons held at near threshold membrane potential showed only low levels of spontaneous synaptic activity (Fig. 2B, left).

The addition of carbachol to the bath perfusion on the hippocampal side of the chamber initiated the rapid appearance of rhythmic field activity in CA3 (in 46 of 84 experiments; 55\%), which was associated with highly synchronized IPSPs in most MS/DB neurons recorded (see below). During hippocampal rhythmic activity, each field event preceded a large IPSP, and the firing rate of the MS/DB neuron increased as IPSPs appeared to pace the intracellular activity. The spiking activity of MS/DB cells typically arose from a depolarizing sag at the end of IPSPs slightly delayed before the next field event as illustrated in Figure 2, $B$ (expanded trace) and $D$.

\section{Characterization of the carbachol-induced synchronized rhythm in CA3 and MS/DB}

Similar to the results obtained with carbachol applications in the isolated intact hippocampus, hippocampal field activity was of large amplitude $(0.40 \pm 0.06 \mathrm{mV})$, appeared with a rapid onset $(1.39 \pm 0.17 \mathrm{~min})$, occurred in episodes at regular rhythmic intervals ( $1-5 \mathrm{~Hz}$ during the peak of the carbachol response), and was of short duration $(2.70 \pm 0.19 \mathrm{~min})$ despite the continuous presence of carbachol. The effect of carbachol was reversible, because similar patterns of both extracellular and intracellular activity could be repeatedly induced after a brief $(\sim 15 \mathrm{~min})$ period of washout (data not shown).

Of $46 \mathrm{MS} / \mathrm{DB}$ neurons recorded during carbachol-induced rhythmic hippocampal activity, 29 neurons (63\%) responded to hippocampal activity with synchronized synaptic potentials. Of those cells, 18 neurons (56\%) showed only IPSPs, whereas the remaining 11 neurons (35\%) received a mixture of prominent IPSPs and smaller EPSPs. All IPSPs in MS/DB followed the field recorded activity in hippocampus, suggesting that the inhibition was caused directly by hippocampal GABAergic input. Accordingly, synchronized rhythmic IPSPs were always reduced in size when the cells were hyperpolarized (see intracellular activity at $V_{\mathrm{m}}-50$ and $-54 \mathrm{mV}$ ) (Fig. $3 \mathrm{C}$ ) and had a reversal potential between -70 and $-80 \mathrm{mV}(n=7)$ (data not shown), suggesting the involvement of $\mathrm{Cl}^{-}$-mediated $\mathrm{GABA}_{\mathrm{A}}$ currents (the predicted $\mathrm{GABA}_{\mathrm{A}}$ reversal potential in our experiments was -80 $\mathrm{mV}$ ). In these experiments, we also took advantage of the separate 
A

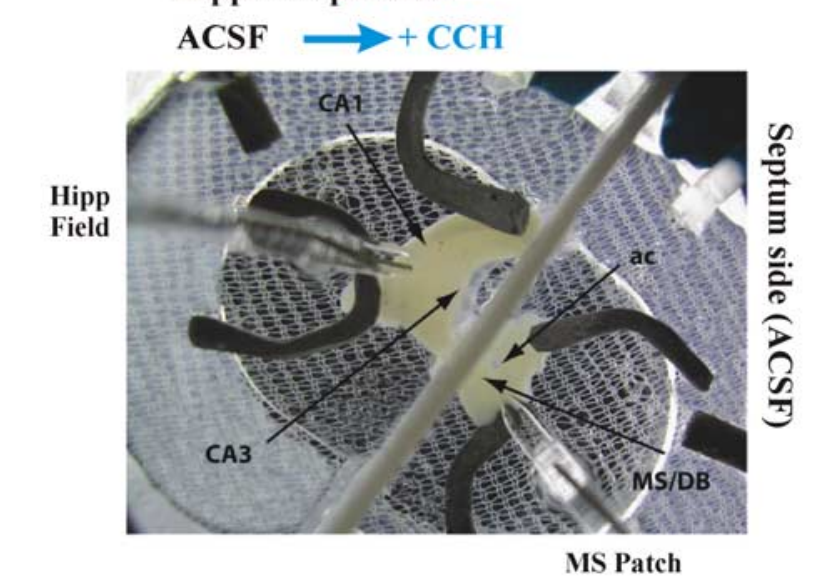

B
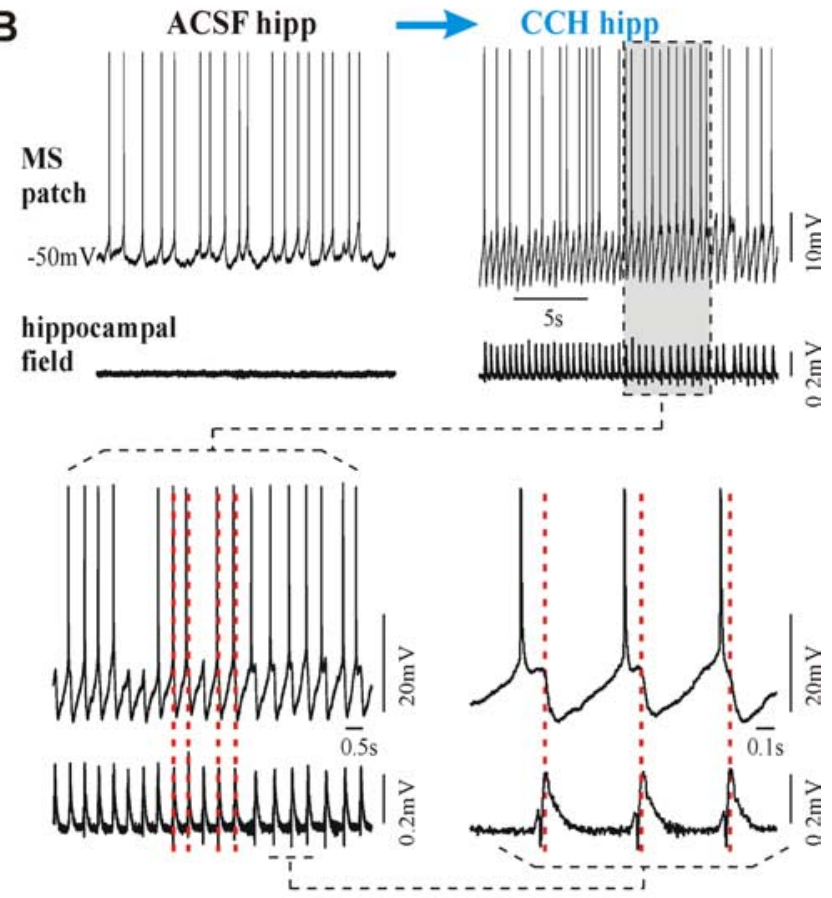

C

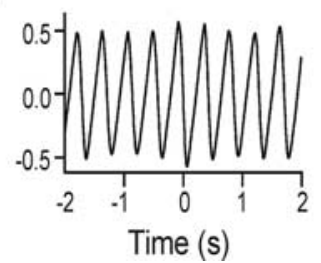

D

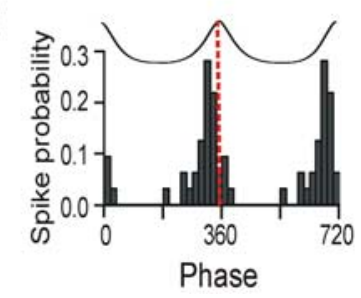

Figure 2. Carbachol infusion in the hippocampus induces rhythmic and synchronized septohippocampal activity. $\boldsymbol{A}$, Low-magnification picture showing the intact septohippocampal preparation in the dual-bath recording chamber and placement of the electrodes. This configuration allowed independent perfusion of the hippocampal compartment (left) with either ACSF or ACSF plus CCH, whereas the septum side (right) was continuously perfused in normal ACSF during concomitant hippocampal field and MS/DB patch recordings. The extracellular field electrode was placed in CA3, and the patch electrode was descended through the MS/DB region. In this preparation, the MS and DBs are readily identifiable by their location with respect to the anterior commissur (ac). B, Typical example of simultaneous recording from an MS/DB neuron (whole-cell, current-clamp; top trace) and from the hippocampus (extracellular field; bottom trace) in the intact septohippocampal preparation. During ASCF perfusion to both hippocampus and septum (left), the MS/DB neuron shows only random discharge and synaptic activity when held at a membrane potential near its firing threshold. After addition of $\mathrm{CCH}(30 \mu \mathrm{m}$; right) to the hippocampal side of the chamber, large rhythmic field potentials appear in CA3, which are

perfusion chamber and found that the application of the $\mathrm{GABA}_{\mathrm{A}}$ receptor antagonist bicuculline $(10 \mu \mathrm{M})$ on the septum side completely blocked synchronized IPSPs $(n=3)$ (data not shown), suggesting the involvement of $\mathrm{GABA}_{\mathrm{A}}$ receptors. Importantly, large rhythmic IPSPs were never observed in MS/DB cells when field activity was absent from the hippocampus. These results are thus consistent with previous anatomical reports that the major projection from the hippocampus to the MS/DB is GABAergic (Toth et al., 1993; Gulyas et al., 2003).

\section{Rhythmic inhibitory input from the hippocampus differentially paces putative GABAergic neurons of the MS/DB}

To determine which type of MS/DB neurons displayed IPSPs in association with rhythmic hippocampal activity, we analyzed the electrophysiological properties of individual cells before carbachol application and sorted them into four categories according to criteria established previously (see Materials and Methods). Briefly, within the sampled population, we found 24 clearly identifiable cells with distinguishing features of the following types: 3 slow-firing, 6 fast- firing, 11 burst- firing, and 4 cluster-firing cells (Table 1). Figure 3 illustrates the typical electrophysiological properties of MS/DB neurons together with the corresponding intracellular activity and simultaneous LFP recordings from CA3 during carbachol-induced hippocampal rhythmic oscillations.

A clear disparity between the different cell types was evident, because most fast- and burst-firing MS/DB neurons fired consistently on the rebound from IPSPs, whereas most slow- and cluster-firing neurons did not (Fig. 4). The fact that spiking of fast- and burst-firing cells was precisely timed after the rebound from IPSPs is furthermore illustrated in Figure 3 (right), where the distribution of IPSPs (green events) and spikes (blue events) in relation to hippocampal field oscillation was plotted as perievent histograms using the peak of CA3 LFPs as a reference for each experiment. Importantly, similar relationships between hippocampal fields, MS/DB IPSPs, and rebound spikes were observed for rhythmic activity at various frequencies even when extracellular oscillations were within the theta range $(5 \mathrm{~Hz})$ (Fig. $3 B$, $C$, fast- and burst-firing cell). Although the number of slowand cluster-firing neurons recorded in these experiments was small ( $n=3$ and 4 , respectively), we found that these cells were generally less responsive to hippocampal activity than the two other groups.

Figure $4 A$ shows the proportion of rebound spikes per IPSPs for the four different types of MS/DB neurons. Burst-firing cells showed the highest proportion of IPSPs with rebound spikes, whereas fast-firing cells displayed a slightly lower amount. In contrast, most slow-firing (cholinergic) and cluster-firing neurons had no rebound spikes or, when present, the lowest number of spikes per IPSPs. The fact that fast- and burst-firing MS/DB neurons fired consistently on the rebound from IPSPs suggested that, paradoxically, hippocampal inhibition may lead to an acti-

\footnotetext{
synchronized with prominent IPSPs and rebound firing in the recorded MS/DB neuron. Note the increased firing rate of the MS/DB neuron during the hippocampal rhythm. Expanded traces from the $\mathrm{CCH}$-induced response are shown below at two different time scales. Vertical dashed lines (red) are positioned at the positive peak of single field events to illustrate how timing was calculated. C, Cross-correlation of the cell potential and hippocampal field during rhythmic activity observed in $\boldsymbol{B}$ indicates a strong synchronization. $\boldsymbol{D}$, A phase histogram shows that the timing of spikes from the neuron in $\boldsymbol{B}$ is relatively phase-locked, because all spikes are clustered near $360^{\circ}$, corresponding to the positive peak of field events. A spike-averaged trace of the hippocampal field activity is shown on top for reference.
} 


\section{A Slow-firing (putative Cholinergic)}

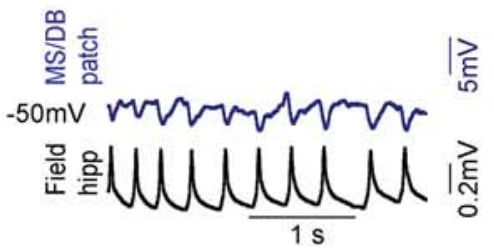

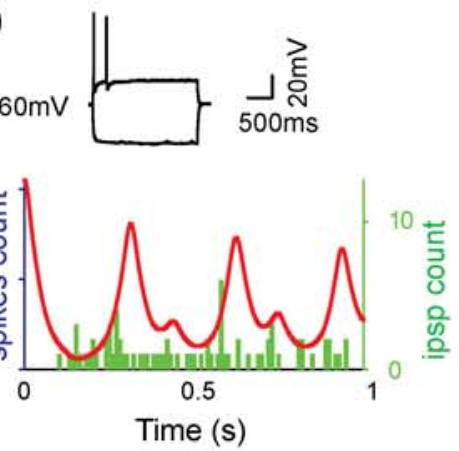

\section{B Fast-firing (putative GABAergic)}
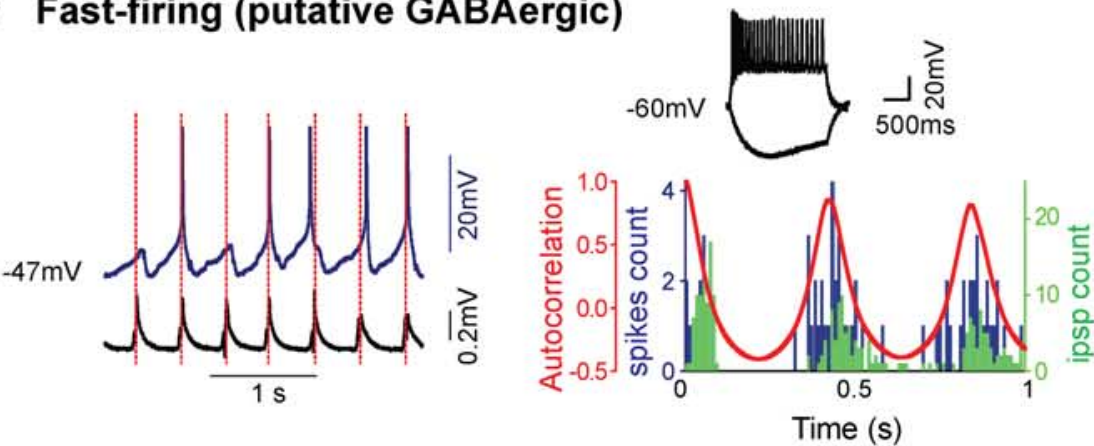

C Burst-firing (putative GABAergic)
$1 \mathrm{~s}$

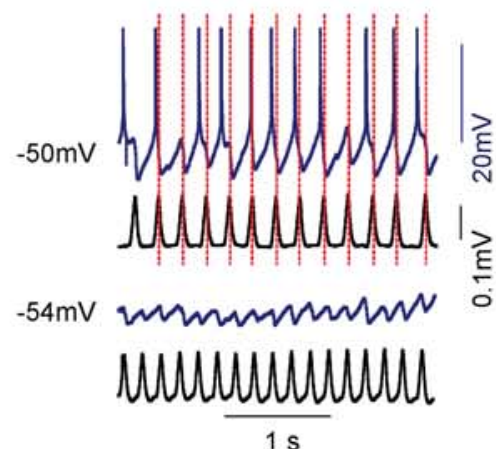

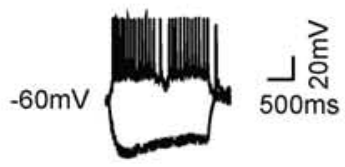

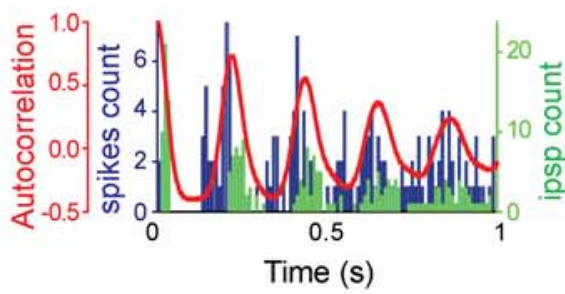

D Cluster-firing (putative Glutamatergic)
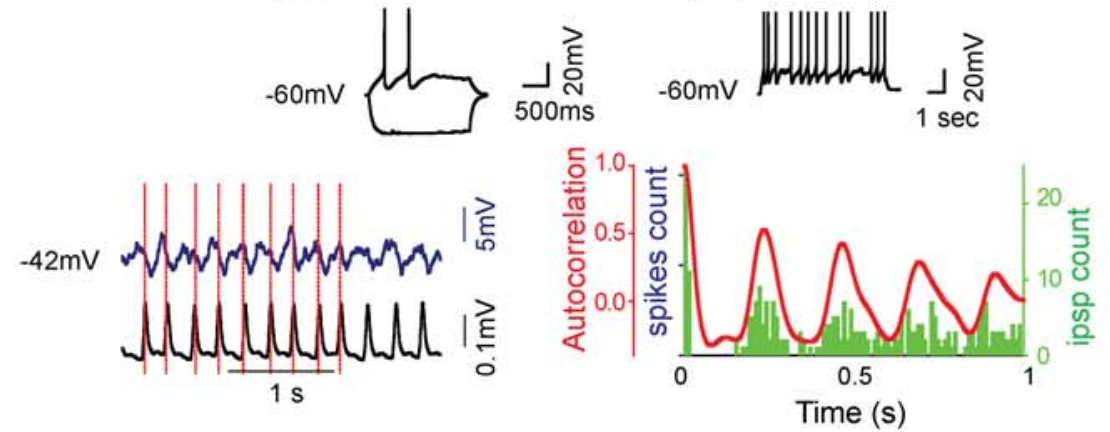

Figure 3. Distinct types of MS/DB neurons show synchronized rhythmic IPSPs and postinhibitory rebound spiking during carbachol-induced hippocampal activity. $\boldsymbol{A}-\boldsymbol{D}$, Electrophysiological characterization (left), carbachol-induced septohippocampal activity (middle), and event cross-correlation histograms (right) showing the timing of IPSPs (green bars) and spikes (blue bars) relative to hippocampal field potential waveform autocorrelation (red) for slow-firing $(\boldsymbol{A})$, fast-firing $(\boldsymbol{B})$, burst-firing $(\boldsymbol{C})$, and cluster-firing $(\boldsymbol{D})$ cells. Note that histogram peaks are distributed at regular intervals, indicating rhythmicity of vation of these two cell types. To investigate this phenomenon in more detail, we specifically examined the firing of MS/DB neurons during the peak of carbachol-induced hippocampal activity by comparing firing frequency during control (1 min) and carbachol peak activity (20 s).

Although, MS/DB neurons were not directly depolarized in response to selective carbachol perfusion on the hippocampal side $\left(V_{\mathrm{m}} \Delta, 0\right)$, further indicating that carbachol was effectively restricted from crossing to the septum side of the chamber, a clear increase in spiking from MS/DB neurons occurred after rhythmic hippocampal activity was initiated with carbachol (Fig. $4 B$ ). We found that in 15 cells ( 1 slow, 6 fast, 7 bursting, and 1 cluster), IPSPs were tightly coupled to rebound activation, increasing by twofold the firing rate of these neurons from $0.6 \pm 0.2$ to $1.2 \pm 0.3 \mathrm{~Hz}(p<0.01)$. Furthermore, most fast- and burst-firing cells were highly activated by hippocampal activity as a large proportion of rhythmic IPSPs ( $\sim 35 \%$ for fast-firing and $55 \%$ for burst-firing) in these cells were followed by a rebound spike, which led to a significant increase in firing rate for both cell types (fast-firing, from $0.2 \pm 0.2$ to $0.6 \pm 0.2 \mathrm{~Hz}$, $p<0.05$; burst-firing, from $1.2 \pm 0.5$ to $1.7 \pm 0.5 \mathrm{~Hz}, p<0.05)$. In contrast, only one of three slow-firing and one of three cluster-firing neurons displayed rebound firing, suggesting that these two cell types may be less susceptible to the excitatory influence of IPSPs from hippocampal drive.

It has been reported that MS/DB neurons in vivo fire bursts of action potentials either in or out of phase with the hippocampal theta rhythm (Stewart and Fox, 1989), a phenomenon recently found to be prominent for GABAergic MS/DB neurons (Borhegyi et al., 2004; Simon et al., 2006). We therefore investigated whether the hippocamposeptal inhibitory input could contribute to the distinct phase preferences of MS/DB neurons. We found that fast- and burst-firing neurons fired at significantly $(p<0.05)$ different phases in relation to

septohippocam pal activity, and that IPSPs are clustered nea the peak of hippocampal field events for fast-, burst-, and cluster-firing cells. As observed in the distribution histograms, spikes are phase locked to the rebounds of the IPSPs for fastand bust-firing MS/DB neurons. Hyperpolarization of the holding potential with current injection in the burst-firing cell reduces the amplitude of IPSPs ( $\boldsymbol{C}$, bottom blue trace) and shows the absence of EPSPS, which could contribute to firing. In the slow-firing $(\boldsymbol{A})$ and cluster-firing $(\boldsymbol{B})$ cells illustrated, IPSPs are less synchronized with fields and do not generate rebound action potentials. All histograms were built from $\sim 30$ s of recordings during the peak of rhythmic septohippocampal activity. 
the peak of the hippocampal field recording (Fig. 4C,D). Indeed, fast-firing neurons fired immediately before the peak (mean $\left.331^{\circ} ; n=4\right)$, whereas burst-firing neurons tended to fire more often between cycles (mean $250^{\circ} ; n=8$ ), indicating that the hippocamposeptal inhibitory input could contribute to differential timing of MS/DB neurons firing. Together, these results suggest that hippocampal inhibition may represent a powerful mechanism for selectively activating and synchronizing large populations of cells in the MS/DB.

Rhythmic hyperpolarization is sufficient to induce rebound spiking in

\section{MS/DB neurons}

The possible intrinsic nature of rebound firing in MS/DB neurons was next examined using short hyperpolarizing pulses, injected rhythmically (at $2 \mathrm{~Hz}$ ), to crudely mimic the effect of hippocampal inhibitory input and test whether this would drive cell spiking. In Figure 5, we show two individual experiments illustrating the distinctive behavior of burst-firing $(A)$ and slow-firing $(B)$ cells during the hyperpolarization protocol. Whereas the rhythmic hyperpolarizations induced prominent rebound activation and an increased firing rate in all burst-firing cells $(n=6)$ and in one of three fast-firing cells (Fig. 5C) (mean increase in firing frequency for those cells, from $0.03 \pm$ 0.01 to $0.7 \pm 0.1 \mathrm{~Hz} ; p<0.05)$, the same protocol produced no change or even a decrease in firing rate for slow- and clusterfiring cells ( $n=4$ and 1, respectively).

This result suggests the presence of intrinsic currents that enable fast- and burst-firing MS/DB neurons to offset rhythmic inhibition with rebound activity. Because both of these classes of neurons are known to express the $I_{h}$ current (Sotty et al., 2003; Morris et al., 2004), which could potentially contribute to their rebound properties, we estimated the size of the depolarizing sag (the $I_{h}$ current-clamp counterpart) of all recorded neurons and found that it was significantly larger in the ones that were driven by hyperpolarizing pulses (Fig. 5D).

\section{Electrical stimulation of the hippocamposeptal GABAergic projections can also synchronize and drive the activity of MS/ DB neurons}

We next went back to examine the mechanisms underlying the apparently paradoxical activation of MS/DB neurons by hippocampal inhibition. Because the carbachol-induced rhythmic activity of the hippocampus was transient and variable, it was not possible to perform adequate pharmacological manipulations in these conditions. As an alternative, compound inhibitory synaptic events were evoked using electrical stimulation of the fornix/ fimbria bundle, which contains the hippocamposeptal fibers that terminate on MS/DB neurons (Toth et al., 1993; Jones et al., 1999) (for illustration of the experimental setup, see Materials and Methods) (Fig. 6A). Evoked IPSPs were similar to the spontaneous ones observed during carbachol-induced hippocampal activity in that their amplitude was reduced when $V_{\text {hold }}$ was hy-
C

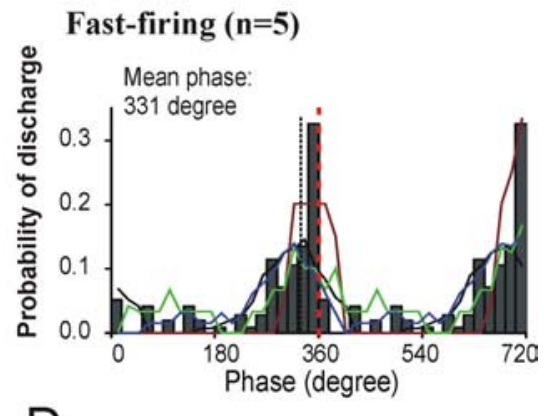

Burst-firing $(\mathrm{n}=9)$

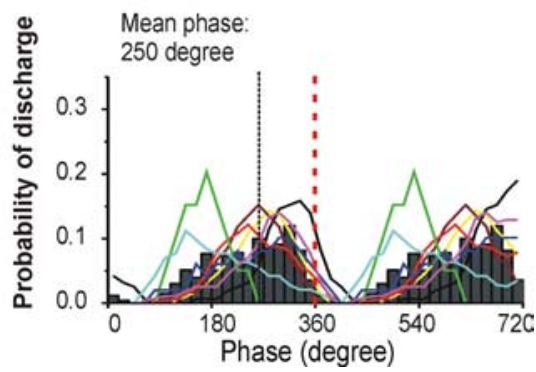

Figure 4. Differential activation and phase locking of identified MS/DB neurons during carbachol-induced hippocampa oscillations. $A, B$, Bar graph showing the proportion of IPSPs followed by rebound spiking during peak CCH-induced ( $)$ and burst-firing $(\boldsymbol{D})$ neurons of the MS/DB. The average probability of discharge for the two different types of was $331^{\circ}$. In contrast, most burst-firing neurons tended to fire action potentials at counter-phase from the hippocampal

perpolarized and also that they were followed by rebound spiking when the cell $V_{\mathrm{m}}$ was near threshold (Fig. $6 \mathrm{~B}$ ). Figure $6 \mathrm{C}$ shows a representative recording of a MS/DB cell held at near threshold membrane potential and firing at low frequency (baseline period). Rhythmic electrical stimulation of the fornix reliably triggered IPSPs throughout the duration of the train and led to increased firing.

We next determined the pharmacological profile of IPSPs driving MS/DB neurons. Evoked IPSPs from MS/DB neurons were completely abolished $5 \mathrm{~min}$ after addition of bicuculline (10 $\mu \mathrm{M})$ to the perfusion bath confirming that they were mediated by $\mathrm{GABA}_{\mathrm{A}}$ receptors $(n=9)$. The effect of bicuculline was slowly reversible after a $>30 \mathrm{~min}$ washout $(n=2)$. In three cells where evoked IPSPs were strongly coupled with rebound activation leading to a $40 \%$ increase in firing frequency (from $0.37 \pm 0.04$ $\mathrm{Hz}$ during baseline to $0.52 \pm 0.05 \mathrm{~Hz}$ during stimulation; $p<$ $0.01)$, blockade of IPSPs with bicuculline completely prevented the stimulation-induced increase in firing rate compared with baseline $(0.35 \pm 0.07 \mathrm{~Hz}$ during baseline vs $0.26 \pm 0.1 \mathrm{~Hz}$ during stimulation; NS) (Fig. 6Di-Diii). This result suggests that IPSPs are critical in mediating the effect of the stimulation on the firing frequency of MS/DB neurons. We next determined the delay in the IPSP onset after the stimulations (Fig. 6E). We found that IPSPs elicited in the slow and fast groups appeared to have more rapid onsets $(30.5 \pm 9.4 \mathrm{~ms}, n=5$ and $51.5 \pm 16.5 \mathrm{~ms}, n=6$, respectively) than those in the burst or cluster groups $(53.2+7.5$ 


\section{A Burst-firing cell}

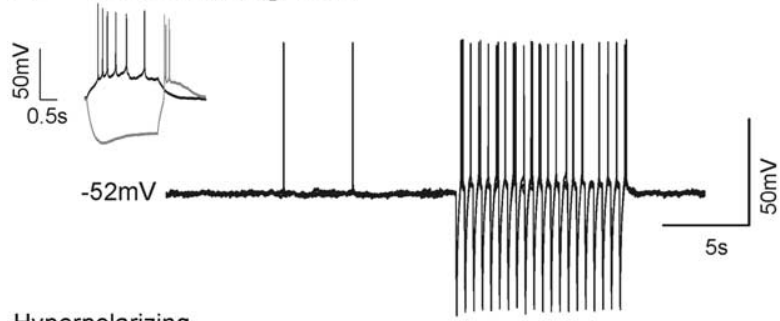

Hyperpolarizing

pulses

bsl

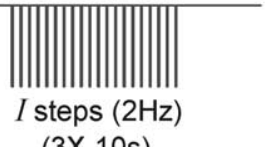

(3X 10s)

\section{B Slow-firing cell}
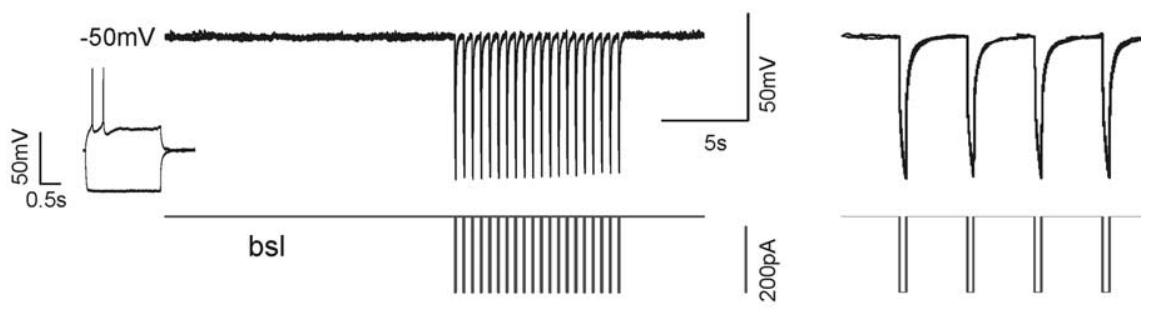

C

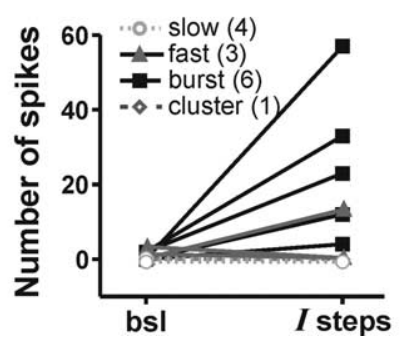

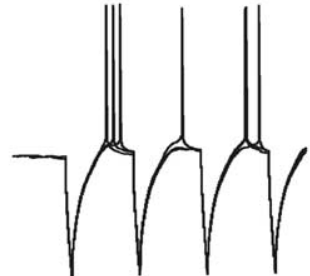
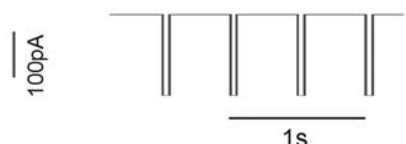

1s
D

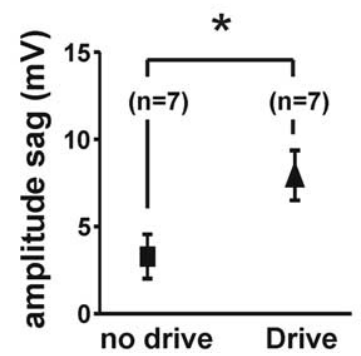

Figure 5. Hyperpolarizing square pulses can elicit rebound spikes and drive rhythmic discharge of fast- and burst-firing MS/DB neurons. $A, B$, Electrophysiological characterization of MS/DB neurons of two types (insets) and illustration of the protocol used to study their response to rhythmic pulses of hyperpolarizing currents steps (left). To establish a baseline level (bsl), MS/DB neurons were first held at a membrane potential near threshold, where they fired at low frequency. Rhythmic trains of hyperpolarizing currents steps (/steps, $2 \mathrm{~Hz}, 10$ pulses of $50 \mathrm{~ms}, 100-200 \mathrm{pA}$ ) were then applied, and the same protocol was repeated three times (traces shown are overlays of the repeated bs I// steps sequence). Burst-firing cells $(\boldsymbol{A})$ fired rapidly after each hyperpolarization (see expanded traces on the right), and this resulted in a reliable rhythmic bursting pattern during the trains. In contrast, repolarization of slow-firing cells ( $\boldsymbol{B}$ ) was slow and did not lead to rebound action potentials. $\boldsymbol{C}$, Scatter plot showing the number of action potentials recorded before (bsl) and during trains of current steps (/ steps) for individual cells of each type $(n=14)$. $\boldsymbol{D}$, Plot comparing the amplitude of the $I_{\mathrm{h}}$-dependent depolarizing sag in cells that did not show increases in firing versus those that did. Error bars represent SEM $\left({ }^{*} p<0.05\right)$.

ms, $n=20$ and $62.2+15.8 \mathrm{~ms}, n=9$, respectively). No statistical significance was noted between the onsets of the four groups.

We then examined whether cell type-specific differences could be observed between electrophysiologically identified neurons. Of $159 \mathrm{MS} / \mathrm{DB}$ neurons recorded, 72 (45\%) displayed IPSPs in response to fornix/fimbria stimulations. When rhythmic trains of stimulations were delivered, 40 of these cells [ 5 of 20 slow-firing (25\%), 6 of 9 fast-firing (67\%), 20 of 31 burst-firing (65\%), and 9 of 12 cluster-firing (75\%)] exhibited IPSP-coupled rebound spiking, which led to an increase in firing frequency compared with baseline level. This increase was significant for fast- and burst-firing neurons, but not for slow- and clusterfiring cells (Fig. 7A). In the latter case, however, it appears that the lack of significance reflected a large variability in the response of cluster-firing cells (some were clearly driven by the stimulations, whereas others were not). Finally, when we analyzed the phase- firing pattern across the two main populations of cells (fast- and burst-firing) that responded to rhythmic stimulation, no difference in the mean phase preference was noted (Fig. $7 B, C$ ), although a few burstfiring cases appeared to fire earlier.

Interplay of IPSPs and $I_{h}$ currents in the rebound activation of MS/DB neurons

The observation that the rebound firing after IPSPs or hyperpolarizing current steps was more prominent in fast- and burstfiring neurons suggested that these cells may have intrinsic properties that facilitate firing in response to rhythmic hippocampal inhibition. A candidate current that is activated during inhibition and is prominent in these two cell types is the hyperpolarization-activated $I_{\mathrm{h}}$ current (Sotty et al., 2003; Morris et al., 2004). We next determined whether low doses of the selective $I_{\mathrm{h}}$ blocker ZD (Harris and Constanti, 1995; Gasparini and DiFrancesco, 1997) could reduce IPSP-mediated rebound activation elicited by phasic fornix/ fimbria stimulation. Four MS/DB cells displaying $I_{\mathrm{h}}$-dependent current (sag, $7.9 \pm$ $1.4 \mathrm{mV}$ ) and firing properties of GABA neurons were recorded in control ACSF and maintained near their firing threshold to provide a low-frequency baseline firing rate. In those cells, rhythmic electrical stimulations evoked IPSPs followed by consistent rebound spikes, which significantly increased the firing rate from $0.1 \pm 0.0 \mathrm{~Hz}$ (during baseline) to $0.5 \pm 0.1 \mathrm{~Hz}$ (during stimulation) (Fig. 8A,D,G). ZD (40 $\mu \mathrm{M})$ was then added to the perfusion medium, and the effect of rhythmic stimulation was tested again after 15 and $30 \mathrm{~min}$. At both time points, ZD reduced the $I_{\mathrm{h}}$-dependent inward rectification (sag) to $-0.1 \pm 0.8$ and $-0.8 \pm 0.8 \mathrm{mV}$, respectively (Fig. $8 \mathrm{H}$ ), and the increase in action potential firing attributable to rebound activation from IPSPs was completely blocked (Fig. $8 B, C, E-$ $G)$. These results suggest that the $I_{\mathrm{h}}$ current plays a crucial role in the inhibition spike-coupling mechanism that allows hippocamposeptal GABAergic projection to selectively control and increase the firing frequency of a group of MS/DB neurons.

\section{Discussion}

This is the first investigation examining in vitro how the hippocampus modulates the MS/DB, which is considered the pacemaker for generating hippocampal theta activity. Oscillations in the hippocampus were elicited with the cholinergic agonist carbachol, an agent well known to sustain network oscillations, including those at theta frequency (McMahon et al., 1998; Fellous and Sejnowski, 2000; Bland and Oddie, 2001). In our experiments, carbachol application to the hippocampus elicited rhythmic oscillations at a frequency between 1 and $5 \mathrm{~Hz}$ in the field, whereas CA3 pyramidal cells occasionally fired phase locked to 
each event. Hence, the carbachol oscillations were useful to elicit single-cell firing of pyramidal neurons in a manner partly similar to that observed during theta in vivo. Our specific aim was to determine whether the rhythmic inhibition originating from hippocamposeptal GABAergic neurons modulates MS/DB neuron firing and to determine how different types of MS/DB neurons (GABAergic, cholinergic, and/or the recently described glutamatergic neurons) were affected. Here, we used an in vitro preparation that consisted of a complete hippocampus and a hemiseptum with intact interconnecting fornix/fimbria pathway. This preparation is very valuable, because the responses specifically generated from hippocampus can be investigated in the MS/DB without the potential confounding effects of multiple interacting rhythm generators known to occur in the intact brain (Vertes and Kocsis, 1997). As expected from previous neuroanatomical data (Toth et al., 1993; Gulyas et al., 2003), we show that inducing rhythmic activity in the hippocampus results in the production of powerful inhibitory potentials in MS/DB neurons. Moreover, the key finding from this study was that the inhibitory potentials could increase the frequency of spiking depending on the cell type and the intrinsic properties of MS/DB neurons. For instance, we show during selective hippocampal rhythmic activity that increases in firing in response to IPSPs occurred prominently in burst- and fast-firing MS/DB neurons (13 of 17 fast and bursting neurons compared with 1 of 3 cholinergic or 1 of 4 cluster-firing putative glutamate neurons). The increased spiking in these cell types was the result of rebound excitation that followed the $\mathrm{GABA}_{\mathrm{A}}$ receptormediated IPSP. The selective increase in rhythmic firing in this population of MS/DB neurons was likely attributable to, in part, their significant expression of $I_{\mathrm{h}}$ current. Because our previous work using single-cell reverse transcription-PCR (Sotty et al., 2003) suggests that such burstand fast-firing neurons are GABAergic, the hippocamposeptal input may promote the rhythmic oscillations of these GABA neurons. In contrast, most slow-firing cholinergic neurons and cluster-firing putatively glutamate neurons that lacked $I_{\mathrm{h}}$ current (Sotty et al., 2003; Garrido-Sanabria et al., 2007) showed rhythmic IPSPs, but very little if any, rebound spiking. Hence, our results propose a model where rhythmic hippocampal oscillations differentially activate MS/DB GABAergic neurons through rhythmic IPSPs but concomitantly promote silence of most cholinergic and glutamatergic neurons. Although IPSPs were prominent in the MS/DB during hippocampal rhythmic oscillations, small EPSPs were also observed in some MS/DB neurons. Because glutamatergic pyramidal and granule cells do not appear to synapse in the MS/DB (Linke et al., 1995) and are likely
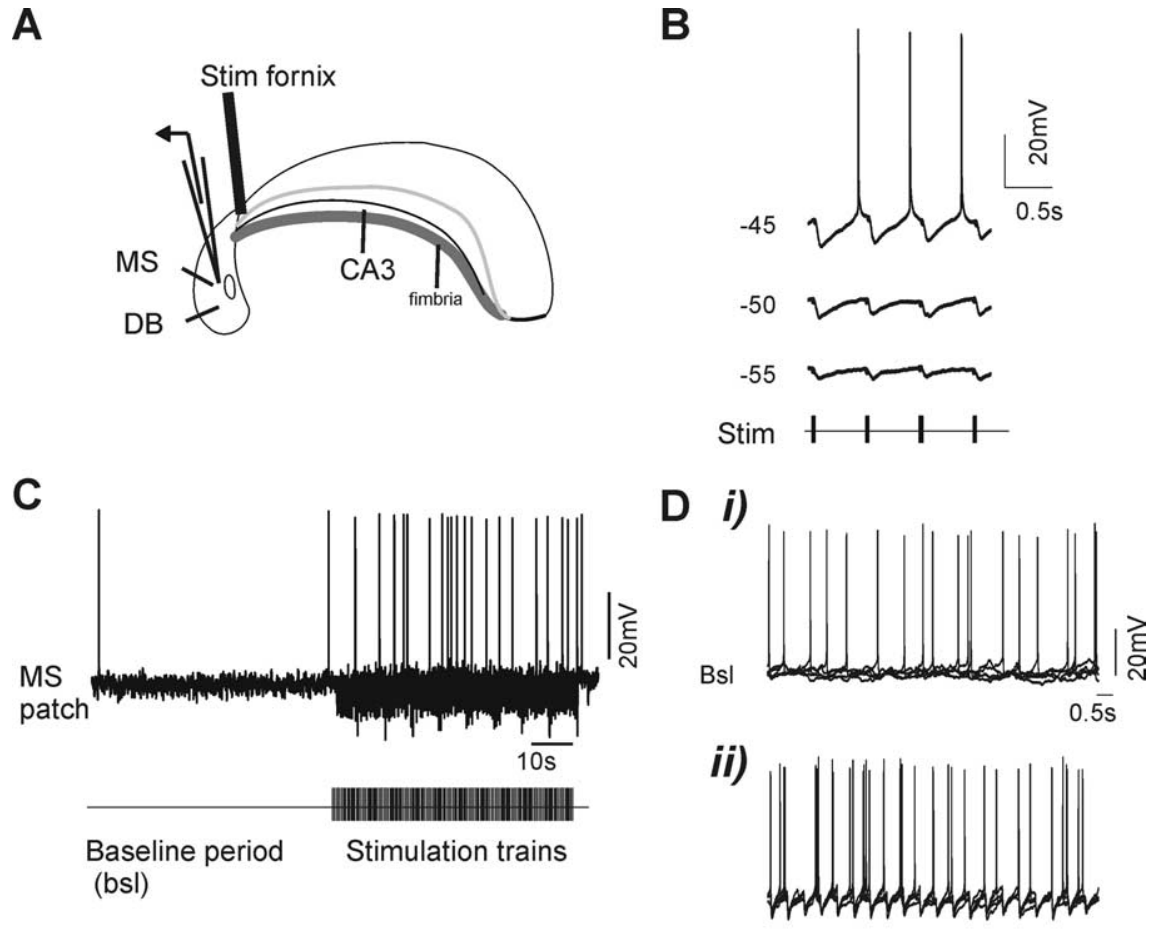

trains $H 1111111111111111$

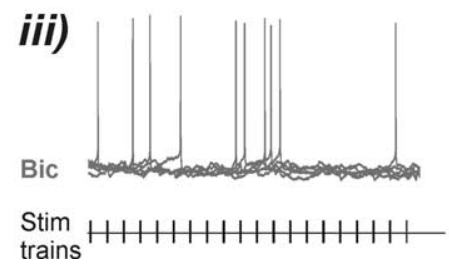

Figure 6. Electrical stimulation of hippocampal GABAergic projections to the septum effectively drives rhythmic bursting activity in MS/DB neurons. $A$, Schematic diagram showing the septohippocampal preparation with placement of the stimulation electrode in the fornix-fimbria bundle (Stim fornix). B, Recording from a burst-firing cell in which brief stimulation trains (5 pulses of $2 \mathrm{~ms} ; 10 \mathrm{~ms} \mid \mathrm{SI}$ ) delivered repeatedly to the fornix evoked short-latency IPSPs followed by rebound action potentials was held at more negative values with tonic current injection. C, Uninterrupted recording from another burst-firing cell illustratmin) at a membrane potential near threshold, a prolonged train of stimulation was applied to the fornix, which evoked large IPSPs leading to an increased firing rate. Di-Diii, Superimposed traces (five times, $10 \mathrm{~s}$ recording episodes) showing spike and $2 \mathrm{~Hz}$ (Stim) (Dii), and the same stimulation protocol applied in the presence of bicuculline (Bic) (Diii). Notice that evoked rhythmic IPSPs elicit rebound spikes increasing the firing rate (compared with baseline) at phase-locked intervals and that both 列 and rebound spiking are abolished after bicuculline (Bic). The membrane potential of this cell was held at a value just above its firing threshold ( $-47 \mathrm{mV}$; determined at the start of experiment). $\boldsymbol{E}$, Spike-triggered average from 10 consecutive evoked IPSPs in ACSF and after addition of bicuculline. For this cell, an onset latency of $20 \mathrm{~ms}$ was measured.

not responsible for generating the EPSPs observed, it is reasonable to assume that they are probably produced by the recently described local glutamatergic MS/DB neurons (Manseau et al., 2005). In fact, cluster-firing MS/DB neurons, which are a class of putatively glutamatergic neurons (Sotty et al., 2003), were occasionally efficiently excited by the IPSPs and sometimes exhibited significant $I_{\mathrm{h}}$. However, the role of these local glutamate MS/DB neurons in synchronizing hippocamposeptal activity remains to be fully investigated.

Our results suggest that GABAergic MS/DB neurons are specially entrained by the rhythmic inhibitory activity from hippocampus. Although these results were obtained when hippocampal oscillations were in the low range of theta frequency 
A
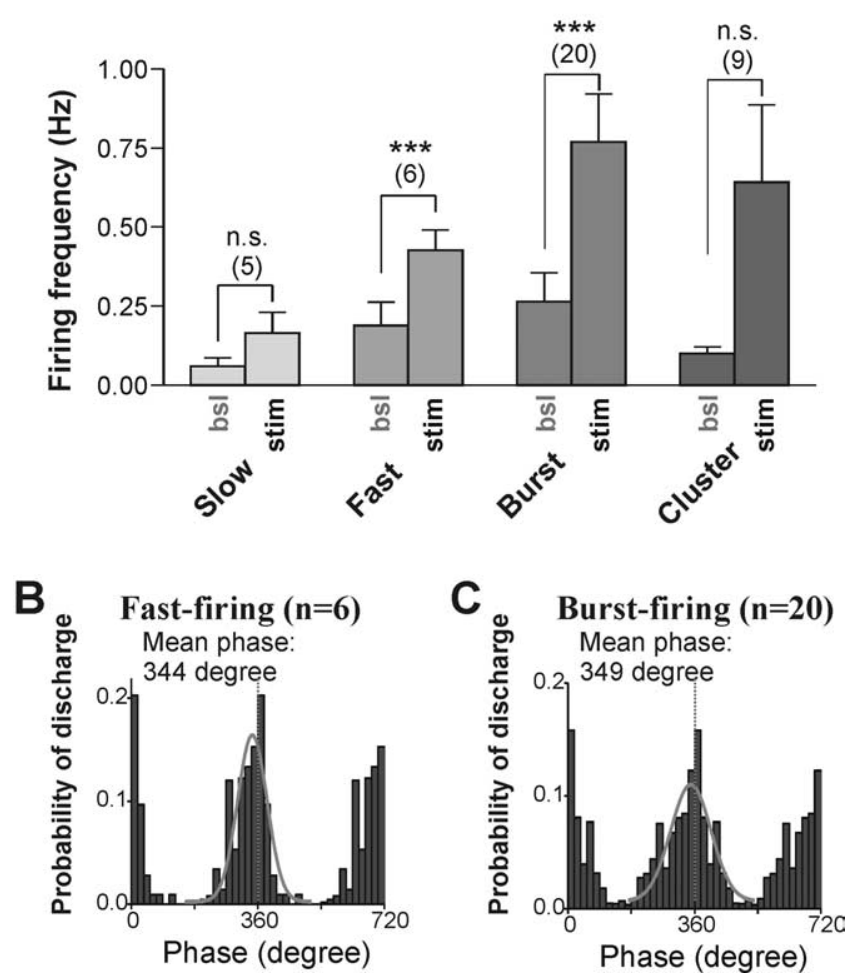

Figure 7. Rhythmic stimulation of fornix/fimbria evoked IPSPs, which significantly activated putative GABAergic neurons of the MS/DB.A, Effect of fornix stimulation (stim) on electrophysiologically identified MS/DB neurons. Compared with baseline level (bsl), the firing of fast- and burst-firing neurons was dramatically increased during rhythmically evoked IPSPS, whereas that of slow- and cluster-firing cells was not changed. Error bars represent SEM ( $\left.{ }^{* * *} p<0.001\right)$. $B, C$, Phase-firing pattern of fast- and burst-firing neurons during rhythmic stimulation of the fornix. The phase histograms show that both fast- and burst-firing neurons exhibit maximal discharge probability just before the following stimulation in each cycle consistent with rebound activation. A Gaussian fit is shown for both distribution (solid gray line), and a dashed vertical line marks the point of stimulation $\left(360^{\circ}\right)$.

(3-5 Hz), it remains to be determined whether higher-frequency theta activity $(6-12 \mathrm{~Hz})$ can produce similar responses in MS/DB neurons. Moreover, it also remains to be established whether carbachol-evoked hippocampal rhythmic oscillations near theta frequency are similar to theta oscillations in vivo (Buzsaki, 2002). Although many have shown theta-like activity using carbachol in slices (Konopacki et al., 1987; Bland and Oddie, 2001), others have suggested that carbachol can elicit hippocampal activity that has some resemblance to epileptiform firing (Williams and Kauer, 1997). However, under our conditions using the septohippocampal preparation, carbachol elicited repetitive oscillatory activity in hippocampus that was clearly nonepileptiform. In fact, CA3 pyramidal cells were not firing in bursts during carbachol oscillations but rather had a high probability to fire one action potential per field event (see firing probability in Fig. 1). It is interesting to note that during theta in vivo, some pyramidal neurons also fire single-action potentials in phase with each theta cycle (Soltesz and Deschenes, 1993; Bland et al., 2005), suggesting that the activity induced by carbachol is useful to mimic some characteristics of theta oscillations. Finally, although we have not investigated directly how hippocamposeptal interneurons behave during carbachol oscillations, a previous study has shown that st. oriens-alveus interneurons (which includes some of the neurons projecting to the septum) fire single spikes phase locked to the field in carbachol and are likely activated by recurrent excitation from pyramidal neurons (McMahon et al., 1998; Hajos et al., 2004). An elegant report recently showed that identified st. oriens-alveus hippocamposeptal neurons also fire at low frequency phase locked to theta in vivo and at a similar phase than pyramidal neurons (Jinno et al., 2007). Together, these reports suggest that hippocamposeptal GABA neurons are active during carbachol or theta oscillations and are phase locked to pyramidal cells.

$I_{\mathrm{h}}$ current is important in hippocamposeptal synchronization We investigated directly whether the hyperpolarization-activated current $I_{\mathrm{h}}$ was involved in hippocamposeptal synchronization. We first showed that phasic electrical stimulation of the fornix/ fimbria pathway (which contains the GABAergic hippocamposeptal fibers) elicited prominent IPSPs in fast- and burst-firing GABAergic MS/DB neurons, thus mimicking the rhythmic inhibition pattern generated during carbachol-induced hippocampal oscillation. Second, we observed a fivefold increase in spiking during the rebound that followed IPSPs, an increase that was blocked by low concentrations of the $I_{\mathrm{h}}$ antagonist ZD7288. These results show that burst- and fast-firing GABAergic MS/DB neurons can resonate in response to the inhibitory rhythmic input from the hippocampus and generate spiking. Because many of these MS/DB GABA neurons are known to project to the hippocampus (Jones et al., 1999; Sotty et al., 2003), they may contribute significantly to the generation and/or modulation of hippocampal theta (Toth et al., 1993). In addition, recent results surprisingly showed that hippocampal theta may be initiated significantly more often in hippocampus than in septum (Nerad and McNaughton, 2006). Therefore, it is possible that local hippocampal theta activity may trigger resonating ( $I_{\mathrm{h}}$ expressing) MS/DB neurons, which in turn modulates and/or helps generate theta across the hippocampus. In relation to the MS/DB and $I_{\mathrm{h}}$, recent studies suggest that specific $I_{\mathrm{h}}$ blockade by ZD7288 infusion in the MS/DB in vivo can significantly affect hippocampal theta activity. Xu et al. (2004) reported that ZD7288 infusion eliminated auditory stimulus-induced hippocampal theta. Although Kocsis and Li (2004) could not replicate the severity of the ZD7288 effects on theta, they found rather that ZD7288 produced a significant reduction (17\%) in the frequency of hippocampal theta in freely moving rats. Noteworthy was their observation that ZD7288 completely eliminated atropine-resistant hippocampal theta during electrical stimulation of the reticular formation (Kocsis and $\mathrm{Li}, 2004$ ). In this respect, their result that $I_{\mathrm{h}}$ in the MS/DB contributes to the atropine-resistant theta in vivo is in line with our finding that $I_{\mathrm{h}}$ is expressed principally in GABAergic neurons and that these cells may make up part of the resistant component.

Interestingly, most MS/DB GABAergic neuron firing is phase locked to hippocampal theta in vivo, and some of these neurons fire at different phases of the cycle (Borhegyi et al., 2004; Simon et al., 2006). In our experiments with carbachol-elicited hippocampal oscillations, we found that burst-firing neurons were more likely to fire near the opposite phase of the oscillation (mean phase, $250^{\circ}$ ), whereas most fast-firing neurons would tend to fire close (mean phase, $330^{\circ}$ ) to the positive peak. However, the variability in the phase of firing was smaller for the fornix/fimbria stimulation experiments, suggesting that mechanisms associated to the network, more than intrinsic properties (i.e., $I_{\mathrm{h}}$ ), are likely implicated. 


\section{Functional relevance to theta}

Although it remains unclear how the hippocampal input to the septum is important in learning, a number of studies by Hasselmo's group and others (Rokers et al., 2002) proposed a model, where the learning dynamics (i.e., encoding and retrieval) in hippocampus are dependent on ACh levels controlled by the hippocampal inhibitory feedback to ACh neurons. Our result showing that hippocampal rhythmic oscillations tend to increase inhibition of MS/DB ACh neurons would also favor a decrease in $\mathrm{ACh}$ levels in hippocampus and is in line with their model. However, the possible differential activation of GABA neurons over ACh neurons will have to be taken into consideration in future studies. Finally, in light of the widely held view that the septum is the pacemaker for hippocampal theta, we propose rather that theta is the result of complex interactions between the septum and hippocampus.

\section{References}

Alonso A, Kohler C (1982) Evidence for separate projections of hippocampal pyramidal and non-pyramidal neurons to different parts of the septum in the rat brain. Neurosci Lett 31:209-214.

Bland BH, Oddie SD (2001) Theta band oscillation and synchrony in the hippocampal formation and associated structures: the case for its role in sensorimotor integration. Behav Brain Res 127:119-136.

Bland BH, Konopacki J, Dyck R (2005) Heterogeneity among hippocampal pyramidal neurons revealed by their relation to theta-band oscillation and synchrony. Exp Neurol 195:458-474.

Blanton MG, Lo Turco JJ, Kriegstein AR (1989) Whole cell recording from neurons in slices of reptilian and mammalian cerebral cortex. J Neurosci Methods 30:203-210.

Blasco-Ibanez JM, Freund TF (1995) Synaptic input of horizontal interneurons in stratum oriens of the hippocampal CA1 subfield: structural basis of feed-back activation. Eur J Neurosci 7:2170-2180.

Borhegyi Z, Varga V, Szilagyi N, Fabo D, Freund TF (2004) Phase segregation of medial septal GABAergic neurons during hippocampal theta activity. J Neurosci 24:8470-8479.

Buzsaki G (2002) Theta oscillations in the hippocampus. Neuron 33:325-340.

Colgin LL, Kubota D, Lynch G (2003) Cholinergic plasticity in the hippocampus. Proc Natl Acad Sci USA 100:2872-2877.

Colom LV, Castaneda MT, Reyna T, Hernandez S, Garrido-Sanabria E (2005) Characterization of medial septal glutamatergic neurons and their projection to the hippocampus. Synapse 58:151-164.

Dragoi G, Carpi D, Recce M, Csicsvari J, Buzsaki G (1999) Interactions between hippocampus and medial septum during sharp waves and theta oscillation in the behaving rat. J Neurosci 19:6191-6199.

Dutar P, Lamour Y, Jobert A (1985) Septohip-
A

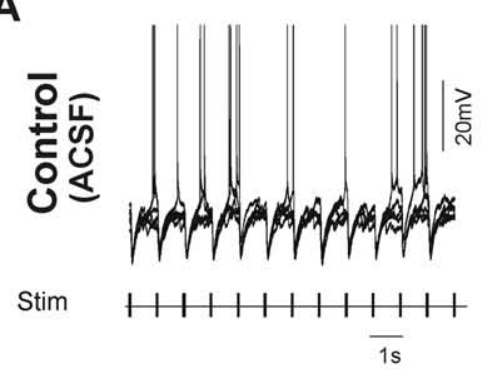

B

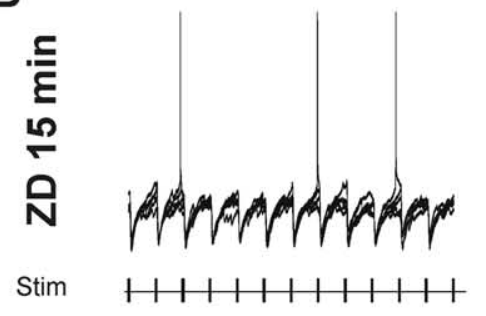

C

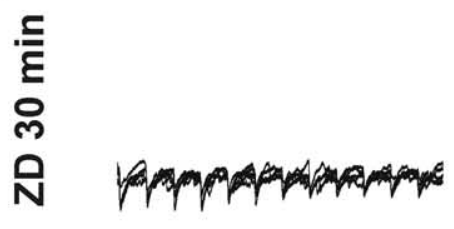

Stim $|1| 1|1| 1|1| 1 \mid$

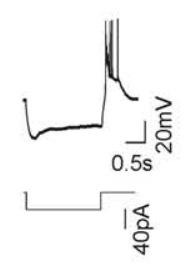

E
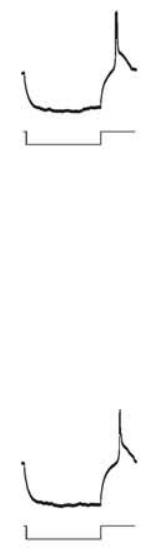

$F$

H

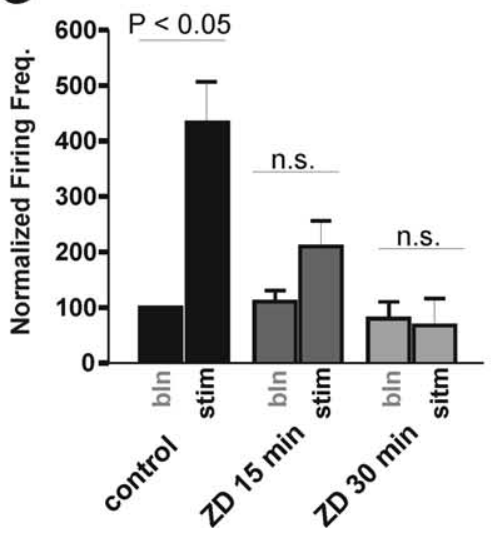

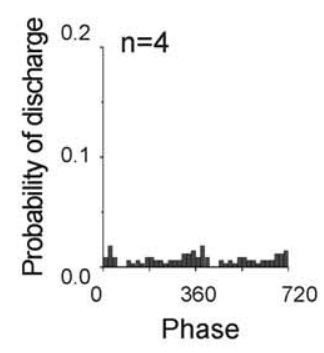
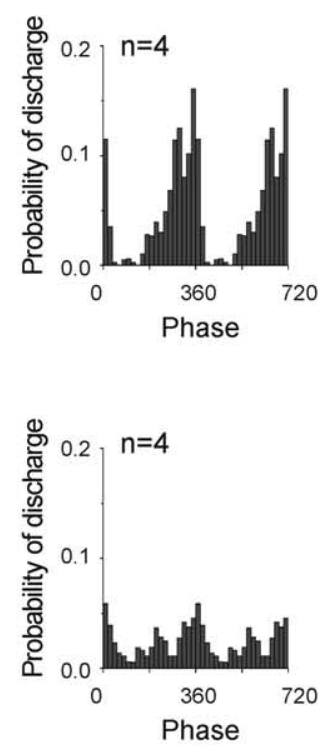

I

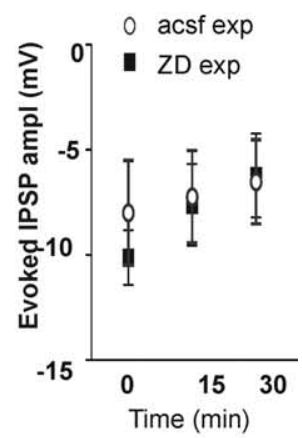

Figure 8. Effect of the $I_{h}$ current blocker $Z D D 288$ (ZD) on rebound spiking of MS/DB neurons. $A$, Trains of electrical stimulation (Stim) to the fornix/fimbria reliably evoked rhythmic IPSPs and rebound spikes in an MS/DB burst-firing neuron during control recording (ACSF perfusion). $\boldsymbol{B}, \boldsymbol{C}$, In contrast, at $15 \mathrm{~min}$ after addition of $Z \mathrm{D}$ to the bath perfusion $(\boldsymbol{B})$, rebound spikes were much sparser, whereas at $30 \mathrm{~min}(\boldsymbol{C})$, they were no longer present, although IPSPs could still be evoked. The insets on the right show the membrane potential response to short hyperpolarizing pulses and the progressive block of $I_{\mathrm{h}}$ current-mediated inward rectification in the recorded neuron after addition of ZD. Scale bars for $\boldsymbol{B}$ and $\mathbf{C}$ are as in $\boldsymbol{A}$. D-F, The phase-firing pattern of MS/DB neurons is altered after ZD. Phase histograms (normalized by total event count during control stimulation) show that the number of rebound spikes as well as their phase coupling decrease with time after ZD application. The number of action potentials per bin (20 bins/cycle) was averaged between four cells, which were all of the burst-firing type for these experiments. G, Bar graph summarizing the effect of $Z D$ on the firing frequency of $M S / D B$ neurons during rhythmic stimulations $(n=4)$. Stimulation produces a large increase in firing frequency compared with baseline (bln) in control condition (ACSF perfusion). This increase is lost, however, at 15 and $30 \mathrm{~min}$ after the beginning of $Z D$ perfusion. $\boldsymbol{H}$, Plot showing the average decrease in the $I_{\mathrm{h}}$-dependent sag after ZD. I, IPSP amplitudes are not significantly changed by ZD. Graph showing that evoked IPSP amplitudes were not significantly changed after ZD (filled square), although they had a slight tendency to decrease gradually in size in a similar manner to those in other MS/DB cells recorded in the absence of ZD (open circles; ACSF control experiments) for the same duration. 
pocampal neurons in the rat: an in vivo intracellular study. Brain Res 340:135-142.

Fellous JM, Sejnowski TJ (2000) Cholinergic induction of oscillations in the hippocampal slice in the slow $(0.5-2 \mathrm{~Hz})$, theta $(5-12 \mathrm{~Hz})$, and gamma (35-70 Hz) bands. Hippocampus 10:187-197.

Fisahn A, Yamada M, Duttaroy A, Gan JW, Deng CX, McBain CJ, Wess J (2002) Muscarinic induction of hippocampal gamma oscillations requires coupling of the $\mathrm{M} 1$ receptor to two mixed cation currents. Neuron 33:615-624.

Freund TF, Antal M (1988) GABA-containing neurons in the septum control inhibitory interneurons in the hippocampus. Nature 336:170-173.

Garrido-Sanabria ER, Perez MG, Banuelos C, Reyna T, Hernandez S, Castaneda MT, Colom LV (2007) Electrophysiological and morphological heterogeneity of slow firing neurons in medial septal/diagonal band complex as revealed by cluster analysis. Neuroscience 146:931-945 .

Gasparini S, DiFrancesco D (1997) Action of the hyperpolarizationactivated current (Ih) blocker ZD 7288 in hippocampal CA1 neurons. Pflügers Arch 435:99-106.

Gorelova N, Reiner PB (1996) Role of the afterhyperpolarization in control of discharge properties of septal cholinergic neurons in vitro. J Neurophysiol 75:695-706.

Griffith WH, Matthews RT (1986) Electrophysiology of AChE-positive neurons in basal forebrain slices. Neurosci Lett 71:169-174.

Gulyas AI, Hajos N, Katona I, Freund TF (2003) Interneurons are the local targets of hippocampal inhibitory cells which project to the medial septum. Eur J Neurosci 17:1861-1872.

Hajos N, Palhalmi J, Mann EO, Nemeth B, Paulsen O, Freund TF (2004) Spike timing of distinct types of GABAergic interneuron during hippocampal gamma oscillations in vitro. J Neurosci 24:9127-9137.

Harris NC, Constanti A (1995) Mechanism of block by ZD 7288 of the hyperpolarization-activated inward rectifying current in guinea pig substantia nigra neurons in vitro. J Neurophysiol 74:2366-2378.

Hasselmo ME, Schnell E (1994) Laminar selectivity of the cholinergic suppression of synaptic transmission in rat hippocampal region CA1: computational modeling and brain slice physiology. J Neurosci 14: 3898-3914.

Jinno S, Klausberger T, Marton LF, Dalezios Y, Roberts JD, Fuentealba P, Bushong EA, Henze D, Buzsaki G, Somogyi P (2007) Neuronal diversity in GABAergic long-range projections from the hippocampus. J Neurosci $27: 8790-8804$.

Jones GA, Norris SK, Henderson Z (1999) Conduction velocities and membrane properties of different classes of rat septohippocampal neurons recorded in vitro. J Physiol (Lond) 517:867-877.

Knapp JA, Morris NP, Henderson Z, Matthews RT (2000) Electrophysiological characteristics of non-bursting, glutamate decarboxylase messenger RNA-positive neurons of the medial septum/diagonal band nuclei of guinea-pig and rat. Neuroscience 98:661-668.

Kocsis B, Li S (2004) In vivo contribution of h-channels in the septal pacemaker to theta rhythm generation. Eur J Neurosci 20:2149-2158.

Konopacki J, MacIver MB, Bland BH, Roth SH (1987) Carbachol-induced EEG "theta" activity in hippocampal brain slices. Brain Res 405:196-198.

Leranth C, Deller T, Buzsaki G (1992) Intraseptal connections redefined: lack of a lateral septum to medial septum path. Brain Res 583:1-11.

Linke R, Pabst T, Frotscher M (1995) Development of the hippocamposeptal projection in the rat. J Comp Neurol 351:602-616.
MacVicar BA, Tse FW (1989) Local neuronal circuitry underlying cholinergic rhythmical slow activity in CA3 area of rat hippocampal slices. J Physiol (Lond) 417:197-212.

Manseau F, Danik M, Williams S (2005) A functional glutamatergic neurone network in the medial septum and diagonal band area. J Physiol (Lond) 566:865-884.

McLennan H, Miller JJ (1974) The hippocampal control of neuronal discharges in the septum of the rat. J Physiol (Lond) 237:607-624.

McMahon LL, Williams JH, Kauer JA (1998) Functionally distinct groups of interneurons identified during rhythmic carbachol oscillations in hippocampus in vitro. J Neurosci 18:5640-5651.

Morris NP, Fyffe RE, Robertson B (2004) Characterisation of hyperpolarization-activated currents $(\mathrm{I}(\mathrm{h}))$ in the medial septum/diagonal band complex in the mouse. Brain Res 1006:74-86.

Nerad L, McNaughton N (2006) The septal EEG suggests a distributed organization of the pacemaker of hippocampal theta in the rat. Eur J Neurosci 24:155-166.

Rokers B, Mercado III E, Allen MT, Myers CE, Gluck MA (2002) A connectionist model of septohippocampal dynamics during conditioning: closing the loop. Behav Neurosci 116:48-62.

Simon AP, Poindessous-Jazat F, Dutar P, Epelbaum J, Bassant MH (2006) Firing properties of anatomically identified neurons in the medial septum of anesthetized and unanesthetized restrained rats. J Neurosci 26:9038-9046.

Soltesz I, Deschenes M (1993) Low- and high-frequency membrane potential oscillations during theta activity in CA1 and CA3 pyramidal neurons of the rat hippocampus under ketamine-xylazine anesthesia. J Neurophysiol 70:97-116.

Sotty F, Danik M, Manseau F, Laplante F, Quirion R, Williams S (2003) Distinct electrophysiological properties of glutamatergic, cholinergic and GABAergic rat septohippocampal neurons: novel implications for hippocampal rhythmicity. J Physiol (Lond) 551:927-943.

Stewart M, Fox SE (1989) Two populations of rhythmically bursting neurons in rat medial septum are revealed by atropine. J Neurophysiol 61:982-993.

Toth K, Borhegyi Z, Freund TF (1993) Postsynaptic targets of GABAergic hippocampal neurons in the medial septum-diagonal band of Broca complex. J Neurosci 13:3712-3724.

Toth K, Freund TF, Miles R (1997) Disinhibition of rat hippocampal pyramidal cells by GABAergic afferents from the septum. J Physiol (Lond) 500:463-474.

Vertes RP, Kocsis B (1997) Brainstem-diencephalo-septohippocampal systems controlling the theta rhythm of the hippocampus. Neuroscience 81:893-926.

Wang XJ (2002) Pacemaker neurons for the theta rhythm and their synchronization in the septohippocampal reciprocal loop. J Neurophysiol 87:889-900.

Williams JH, Kauer JA (1997) Properties of carbachol-induced oscillatory activity in rat hippocampus. J Neurophysiol 78:2631-2640.

Winson J (1978) Loss of hippocampal theta rhythm results in spatial memory deficit in the rat. Science 201:160-163.

Xu C, Datta S, Wu M, Alreja M (2004) Hippocampal theta rhythm is reduced by suppression of the H-current in septohippocampal GABAergic neurons. Eur J Neurosci 19:2299-2309. 